\title{
Optical properties of dynamical axion backgrounds
}

\author{
Jamie I. McDonald ${ }^{1, *}$ and Luís B. Ventura $\odot^{2, \dagger}$ \\ ${ }^{1}$ Physik-Department, James-Franck-Straße, Technische Universität München, 85748 Garching, Germany \\ ${ }^{2}$ Departamento de Física da Universidade de Aveiro and CIDMA, \\ Campus de Santiago, 3810-183 Aveiro, Portugal
}

(Received 20 March 2020; accepted 22 May 2020; published 4 June 2020)

\begin{abstract}
We discuss spectral distortions, time delays, and refraction of light in an axion or axion-plasma background. This involves solving the full set of geodesic equations associated to the system of Hamiltonian optics, allowing us to self-consistently take into account the evolution of the frequency, momentum, and position of photons. We support our arguments with analytic approximations and full numerical solutions. We also describe both nonintegrated and integrated versions of these effects, the latter of which depends on the whole history of the photon trajectory through the axion background. Remarkably, the introduction of a plasma enhances the sensitivity to axion-induced optical phenomena, allowing chiral refraction, integrated time delays, and integrated frequency shifts to occur at first order in the axion-photon coupling. This suggests a general enhancement of many axion-induced dispersive effects when the background refractive index is different from 1.
\end{abstract}

DOI: 10.1103/PhysRevD.101.123503

\section{INTRODUCTION}

Axions remain promising candidates for beyond the Standard Model physics. The QCD axion emerges as a solution to the strong- $C P$ problem [1-3], while string theory extensions of the Standard Model predicate a plethora of axionlike particles [4,5]. Axions could also solve the dark matter problem [6-10]. The masses of axions can span a large range of scales, with Compton wavelengths on the order of galaxies down to table-top sizes of millimeters and below. As a result, the axion is the subject of many current and proposed laboratory searches [11-19].

The aim of the present work is to explore the optical properties of an axion background due to its coupling to photons

$$
\mathcal{L}_{a \gamma \gamma}=-\frac{g_{a \gamma \gamma}}{4} a F_{\mu \nu} \tilde{F}^{\mu \nu},
$$

where $g_{a y \gamma}$ is the axion-photon coupling, $a$ is the axion field, and $F_{\mu \nu}$ and $\tilde{F}_{\mu \nu} \equiv \epsilon_{\mu \nu \rho \sigma} F^{\rho \sigma} / 2$ are the photon field strength and its dual, respectively. This interaction leads to nontrivial dispersion in axion backgrounds and, since it violates parity, leads to birefringence. This can generate a

\footnotetext{
jamie.mcdonald@tum.de

†lbventura@ua.pt
}

Published by the American Physical Society under the terms of the Creative Commons Attribution 4.0 International license. Further distribution of this work must maintain attribution to the author(s) and the published article's title, journal citation, and DOI. Funded by SCOAP ${ }^{3}$. relative phase velocity splitting between left and right polarized light, leading to Faraday-like rotation of linearly polarized light [20-23]. Modifications to the group velocity were considered in Ref. [24], where it was suggested this could lead to time delays for axion profiles around pulsars. It was also proposed that axion backgrounds could lead to polarization-dependent bending of light [25]. However, a more systematic analysis of photon geodesics revealed this could not happen at $\mathcal{O}\left(g_{\text {ary }}\right)$ [26] for a pure axion background.

In fact, the authors of Ref. [26] made a very important and general observation. While there are many references which deal with the propagation of light through an axion background [20,25,27-30], these are, in general, not fully realistic since they neglect either the time or space dependence of the axion background, which both affect photon propagation. For example, in this work, we show that an axion background localized along the line of sight with space and time dependence leads to integrated effects, dependent on the whole history of the trajectory, including frequency shifts, time delays, and refraction. This is reminiscent ${ }^{1}$ of the integrated and nonintegrated Sachs-Wolfe effects [31].

As the axion background is both time and space dependent, frequency and momentum are no longer conserved quantities, and a proper discussion of dispersion must self-consistently take into account variations of these and the position along the ray path. This can be achieved by deriving a system of Hamiltonian optics equations [32] whose solutions give the photon geodesics and capture all

\footnotetext{
${ }^{1}$ We thank David J. E. Marsh for pointing this out.
} 
the necessary details about group dispersion, frequency and momentum shifts, time delays, and refraction. Furthermore, these equations allow one to study general axion backgrounds without the need to assume any hierarchy between temporal and spatial gradients as would happen for nonrelativistic backgrounds.

The present paper is concerned with examining these key observables within the optics equations, extending the discussion of Ref. [26] by accounting for higher-order effects in $g_{a \gamma \gamma}$, modifying the background refraction index by the presence of a background plasma and studying a wider class of observable effects.

First, we point out the difference between leading and higher-order corrections to the photon frequency/ momentum. We show that, when plasma is present, one can have integrated frequency shifts even for localized axion profiles which vanish at the end points of the photon trajectory. By contrast, in the absence of the plasma, at first order in $g_{a \gamma \gamma}$, there is no integrated frequency shift, consistent with Ref. [26]. Instead, the asymptotic (integrated) frequency shift in the plasma-free case occurs at order $g_{a \gamma \gamma}^{2}$.

Second, we demonstrate polarization-dependent group dispersion at first order in $g_{\text {ary }}$ for background refractive indices different from $1, n_{0} \neq 1$, provided here by plasma. We show that this leads to polarization-dependent time delays at $\mathcal{O}\left(g_{\text {ary }}\right)$. We also discuss higher-order group velocity corrections at $\mathcal{O}\left(g_{a \gamma \gamma}^{2}\right)$ and discuss these in terms of group versus signal velocities, relevant for the speed of information transmission via pulses [33].

Third, we confirm that there is no asymptotic bending of light at $\mathcal{O}\left(g_{\text {ary }}\right)$ when the background refractive index is equal to $1, n_{0}=1$ [26], but show that birefringent refraction does happen at $\mathcal{O}\left(g_{\text {ary }}\right)$ when $n_{0} \neq 1$.

The structure of the paper is as follows. In Sec. II, we derive the dispersion relations for photons in a general axion background with an additional plasma component. Here, we set up the geodesic equations and draw an analogy with Hamiltonian perturbation theory as an interpretation of our perturbative expansion. In Secs. III and IV, we study a simple $1+1$-dimensional axion background, which is sufficient to illustrate frequency shifts, group dispersion, and time delays. Then, in Sec. V, we work in $2+1$ dimensions, which allows us to demonstrate refraction within a plasma at $\mathcal{O}\left(g_{\text {ary }}\right)$ and without a plasma at

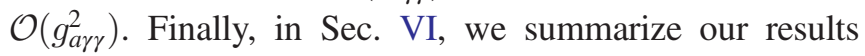
and speculate on some applications and proposals for future work. We have used natural Lorentz-Heaviside units $c, \epsilon_{0}, \mu_{0}=1$ in this work.

\section{A. Physical motivation}

The work presented here can be used as a general toolkit which, when appropriately applied and extended, offers the potential to probe the existence of axion backgrounds in a variety of ways. There are, in particular, two noteworthy optical features of axion backgrounds: first, that they violate parity by generating polarization-dependent dispersion and, second, that the observed frequency shifts, varying arrival time, and refraction will undergo periodic modulation due to the oscillating axion field. The following aspects are relevant for a comprehensive discussion of axion backgrounds:

(1) Axion profiles. There are, at least, two interesting types of axion backgrounds to which our results might be applied. One is to postulate axion dark matter, either as a virialized QCD axion background of particles or as an ultralight scalar whose Compton wavelength is of galactic sizes [10]. Both of these will lead to birefringent signals with a period multiple of $2 \pi / m_{a}$. Another possibility is to use a superradiant black hole background [34-36], where the axion field values can be especially high. For this case, our discussion of optical properties of distant sources, which rely on integrated effects, are particularly relevant.

(2) Time delays. Time delays from a galactic/ astrophysical axion background could possibly be probed via precision pulsar timing, see, e.g., Ref. [37], although here they arise through a direct coupling of the axion field to the photon, rather than via geometric distortions. See also Refs. [38,24]. Equally, a local axion background could be studied in a terrestrial timing experiment, possibly via interferometry [22].

(3) Spectral shifts. Our analysis of integrated spectral distortions from axion backgrounds along all or part of the line of sight suggests that, for a line signal from an astrophysical source, one would observe a periodic shift in the position of the peak frequency. For a linearly polarized signal, a double image in frequency space for each polarization would be produced, with the two peaks experiencing periodic oscillation. This could be due-as with time delays - to a dark matter background. Another possibility is to observe the spectrum of a superradiant black hole accretion disk whose emission passes through the axion background. In both cases, the spectrum would be shifted periodically, with lower frequencies experiencing a greater magnitude of shifting. One could also hope to observe the

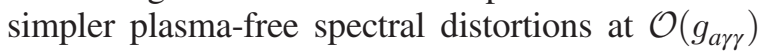
in, e.g., a terrestrial experiment as suggested in Ref. [26] where one measures the frequency changes between emission and detection.

(4) Refraction. Our results show that birefringent re-

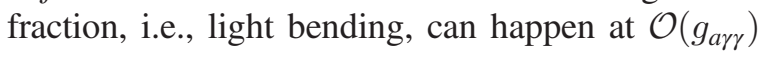
when there is a nontrivial background refractive index $n_{0}$, here supplied by a plasma. The scenario of 
polarization-dependent refraction by a superradiant black hole was considered in Ref. [25], but ruled out at leading order in $g_{a \gamma \gamma}$ by a more detailed analysis [26] of the optics equations. However, in light of our results, it could be interesting to rerun the analysis of Ref. [25] with a plasma component from an accretion disk or interstellar medium and compare the refraction angles to resolutions of upcoming and current telescopes.

\section{DISPERSION IN AXION BACKGROUNDS}

The term (1) leads to the following modification of Maxwell's equations,

$$
\begin{gathered}
\nabla \cdot \mathbf{E}=\rho-g_{a \gamma \gamma} \mathbf{B} \cdot \nabla a, \\
\nabla \times \mathbf{B}-\dot{\mathbf{E}}=\mathbf{J}+g_{a \gamma \gamma} \dot{a} \mathbf{B}+g_{a \gamma \gamma} \nabla a \times \mathbf{E}, \\
\nabla \cdot \mathbf{B}=0, \\
\dot{\mathbf{B}}+\nabla \times \mathbf{E}=0,
\end{gathered}
$$

where $\mathbf{E}$ and $\mathbf{B}$ are the electric and magnetic fields, $\mathbf{J}$ and $\rho$ are current and charge densities, and the dots represent time derivatives. The electromagnetic fluctuations are related to current fluctuations via

$$
\mathbf{J}=\sigma \cdot \mathbf{E},
$$

where $\sigma_{i j}$ is a conductivity tensor. Throughout, we assume an isotropic and collisionless medium for which the conductivity at a given frequency takes the form $\sigma_{i j}(\omega)=$ $i \delta_{i j} \omega_{p}^{2} / \omega$ where $\omega_{p}^{2}=n_{\mathrm{cc}} e^{2} / m_{\mathrm{cc}}$ is the plasma frequency squared and $n_{\mathrm{cc}}$ is the number density of charge carriers. This could arise, for instance, from the interstellar medium, the companion star of a black hole, or perhaps an accretion disk, though the following discussion remains general. One could also keep in mind the possibility of any medium which endows the photon with a nontrivial refractive index, which achieves the same end. This may be relevant in exploring extensions of the following analysis to laboratory setups.

With some straightforward manipulations of Maxwell's equations, we obtain

$$
\begin{gathered}
\square \mathbf{E}+\nabla(\nabla \cdot \mathbf{E})+\sigma \dot{\mathbf{E}}+g_{a \gamma \gamma} \frac{d}{d t}[\dot{a} \mathbf{B}+\nabla a \times \mathbf{E}]=0, \\
\square \mathbf{B}+\sigma \dot{\mathbf{B}}-g_{a \gamma \gamma} \nabla \times[\dot{a} \mathbf{B}+\nabla a \times \mathbf{E}]=0 .
\end{gathered}
$$

The first equation is derived by taking the time derivative of Eq. (3) and inserting (5) to eliminate $\dot{\mathbf{B}}$. The second equation follows by taking the time derivative of (5) and using (3) to eliminate $\dot{\mathbf{E}}$. Next, we use the geometrical optics approximation, defined by the requirement that the photon wavelength should be smaller than the gradient scales of the axion background. This amounts, schematically, to the limit $\partial_{\mu} a / a \ll \partial_{\mu} \mathbf{E} / \mathbf{E}, \partial_{\mu} \mathbf{B} / \mathbf{B}$. This requirement allows us to consider solutions of the form

$$
\mathbf{E}=\mathbf{E}_{0} e^{i S}, \quad \mathbf{B}=\mathbf{B}_{0} e^{i S},
$$

where frequency and momentum are identified as $\omega=-\dot{S}$ and $\mathbf{k}=\nabla S$. We derive dispersion relations by neglecting further derivatives of $\omega, \mathbf{k}$ and $\mathbf{E}_{0}, \mathbf{B}_{0}$. This is the standard eikonal approximation, which leads to local dispersion relations from which optics equations are derived. With this, we can use charge conservation $\dot{\rho}+\nabla \cdot \mathbf{J}=\dot{\rho}+\sigma \nabla$. $\mathbf{E}=0$ to express

$$
\nabla \cdot \mathbf{E}=-\left(1-\frac{\omega_{p}^{2}}{\omega^{2}}\right)^{-1} g_{a \gamma \gamma} \mathbf{B} \cdot \nabla a
$$

and use the latter to eliminate the $\nabla(\nabla \cdot \mathbf{E})$ term in (7),

$$
\square \mathbf{E}+\omega_{p}^{2} \mathbf{E}-g_{a \gamma \gamma} \frac{(\nabla \mathbf{B}) \cdot \nabla a}{1-\omega_{p}^{2} / \omega^{2}}+g_{a \gamma \gamma}[\dot{a} \dot{\mathbf{B}}+\nabla a \times \dot{\mathbf{E}}] \simeq 0,
$$

$$
\mathbf{B}+\omega_{p}^{2} \mathbf{B}-g_{a \gamma \gamma}[\dot{a} \nabla \times \mathbf{B}+\nabla a(\nabla \cdot \mathbf{E})-(\nabla a \cdot \nabla) \mathbf{E}] \simeq 0
$$

where we have neglected second derivatives of the axion field in accordance with the geometric optics limit described above. Upon using (9), this system can then be written as $[26,32]$

$$
\mathbf{M}(\omega, \mathbf{k}) \cdot(\mathbf{E}, \mathbf{B})^{T}=0,
$$

where $\mathbf{M}$ is a matrix whose structure can be read from Eqs. (11) and (12). The condition (13) is equivalent to demanding that an eigenvalue of $\mathbf{M}$ must vanish, which gives the dispersion relation of a particular mode. Explicitly, the eigenvalues of (11) and (12) involving the axion-photon coupling are given by

$$
\begin{aligned}
D^{ \pm}= & k^{2}-\omega_{p}^{2} \pm \frac{1}{\left[\omega^{2}-\omega_{p}^{2}\right]^{1 / 2}}\left[\omega^{2} g_{a \gamma \gamma}^{2}\left((k \cdot \partial a)^{2}-k^{2}(\partial a)^{2}\right)\right. \\
& \left.+\omega_{p}^{2} g_{a \gamma \gamma}^{2}\left(\dot{a}^{2} k^{2}-2 \dot{a} \omega(k \cdot \partial a)+(\partial a)^{2} \omega^{2}\right)\right]^{1 / 2},
\end{aligned}
$$

where $k^{\mu}=(\omega, \mathbf{k})$ and $k^{2}=k_{\mu} k^{\mu}$ and $\partial a \equiv \partial_{\mu} a=$ $(\dot{a}, \nabla a)$. In the plasma-free case, we recover the well-known dispersion relation (by setting $D^{ \pm}=0$ ) of Refs. [20,25]

$\omega_{p}=0: \quad k^{2}= \pm g_{a \gamma \gamma}\left[(k \cdot \partial a)^{2}-k^{2}(\partial a)^{2}\right]^{1 / 2}$. 
This is the standard form of eigenvalues found for a Chern-Simons-like interaction considered in many works [27-29] and references therein. Note that, in the present work, we assume a sufficiently weak axion field value so that the photon never suffers a tachyonic instability [28]. However, it is worth mentioning that, for sufficiently low momenta and high density axion fields, this could have interesting physical implications [39]. We speculate more on these in the discussion.

In many of these references-especially for instance the classic one [20] - the vector $\partial_{\mu} a$ was treated as a constant background quantity in the spirit of Lorentz and $C P T$ violation. However, recently, the authors of Ref. [26] made a very important observation, namely that when the axion field $a$ is dynamical, the phase space trajectories themselves are altered by temporal and spatial gradients. Due to the dynamical nature of the background, the frequency and momentum of the light ray are no longer conserved quantities, and therefore need to be evolved, along the its trajectory, by solving the full set of Hamiltonian optics equations, along the lines of Ref. [32]. To set up the system of equations, one first notes that to enforce dispersion relations along rays, the eigenvalues $D^{ \pm}$must vanish everywhere along trajectories, which implies

$$
\frac{d D^{ \pm}}{d \tau}=\frac{\partial D^{ \pm}}{\partial k^{\mu}} \frac{d k^{\mu}}{d \tau}+\frac{\partial D^{ \pm}}{\partial x^{\mu}} \frac{d x^{\mu}}{d \tau}=0
$$

where $\tau$ is an arbitrary worldline parameter. We then define trajectories according to [32]

$$
\frac{d x^{\mu}}{d \tau} \equiv-\frac{\partial D^{ \pm}}{\partial k_{\mu}}, \quad \frac{d k^{\mu}}{d \tau} \equiv \frac{\partial D^{ \pm}}{\partial x_{\mu}},
$$

where $k^{\mu}=(\omega, \mathbf{k})$ and $k_{\mu}=(\omega,-\mathbf{k})$. These are simply generalizations of Hamilton's equations. One can then eliminate $\tau$ and instead use $t$ as a worldline parameter to arrive at

$$
\begin{aligned}
& \frac{d \mathbf{x}}{d t}=-\frac{\partial D^{ \pm} / \partial \mathbf{k}}{\partial D^{ \pm} / \partial \omega}=\frac{\partial \omega}{\partial \mathbf{k}}, \\
& \frac{d \mathbf{k}}{d t}=\frac{\partial D^{ \pm} / \partial \mathbf{x}}{\partial D^{ \pm} / \partial \omega}=-\frac{\partial \omega}{\partial \mathbf{x}}, \\
& \frac{d \omega}{d t}=-\frac{\partial D^{ \pm} / \partial t}{\partial D^{ \pm} / \partial \omega}=\frac{\partial \omega}{\partial t} .
\end{aligned}
$$

In fact, one notes that all these equations take the form

$$
\frac{d f}{d t}=\{f, \omega\}+\partial_{t} f, \quad f=\omega, \mathbf{k}, \mathbf{x},
$$

where $\{A, B\}=\partial_{\mathbf{x}} A \partial_{\mathbf{k}} B-\partial_{\mathbf{k}} A \partial_{\mathbf{x}} B$ is the Poisson bracket. The interpretation of these equations becomes immediately transparent: they are precisely a system of Hamilton-Jacobi equations where $\omega=\omega(\mathbf{x}, \mathbf{k} ; t)$ can be seen as a one-particle, time-dependent Hamiltonian yielding the particle energy and $\mathbf{x}$ and $\mathbf{k}$ can be seen as the standard canonical position and momentum variables. We can think of $\omega_{0}(\mathbf{x}, \mathbf{k}, t) \equiv \sqrt{|\mathbf{k}|^{2}+\omega_{p}^{2}}$ as the (unperturbed) Hamiltonian, which is then modified by the axion interaction. This can be treated formally by using Hamilton-Jacobi perturbation theory, constructing a perturbative expansion (in powers of $g_{a \gamma \gamma}$ ) about the zerothorder solutions

$$
\begin{gathered}
\omega_{0}^{2}=\mathbf{k}_{0}^{2}+\omega_{p}^{2}, \\
\mathbf{x}_{0}(t)=\mathbf{v}_{g}^{0} t+\mathbf{x}_{i}, \\
\mathbf{v}_{g}^{0}=\mathbf{k}_{0} / \omega_{0} .
\end{gathered}
$$

In Ref. [26], the authors kept only terms of $\mathcal{O}\left(g_{\text {ary }}\right)$ in this expansion. In what follows, we discuss higher-order corrections which reveal a number of interesting properties. Since the dispersion relation (14) is, in general, quite complicated and involves finding the roots of a sixth-order polynomial, we will analyze some special cases where $\omega=\omega(\mathbf{k})$ takes a simpler form and, can be solved exactly.

\section{SPECTRAL DISTORTIONS}

One notable case for which the optics equations are easily tractable and the dispersion relation is simple is a $1+1$-dimensional background with

$$
a=a(t, x), \quad \text { and } \quad \mathbf{k} \| \nabla a,
$$

so the photon momentum $\mathbf{k}$ is always parallel to the axion gradients $\nabla a$. We can then choose coordinates such that

$$
\mathbf{k}(t)=(k(t), 0,0), \quad \mathbf{x}(t)=(x(t), 0,0) .
$$

In this case, $D_{ \pm}$is

$$
D^{ \pm}(t, x)=\omega^{2}-k^{2}-\omega_{p}^{2} \pm g_{a \gamma \gamma}\left(k \dot{a}+\omega a^{\prime}\right),
$$

where primes denote differentiation with respect to $x$. The exact dispersion relation is then straightforward:

$\omega(k)=\left[k^{2}+\omega_{p}^{2} \mp g_{a \gamma \gamma} k \dot{a}+\frac{g_{a \gamma \gamma}^{2} a^{\prime 2}}{2}\right]^{1 / 2} \mp \frac{g_{a \gamma \gamma} a^{\prime}}{2}$.

This case is particularly interesting since it allows us to study the passage of light rays through an axion background localized in space. Furthermore, it is sufficient to study group dispersion, time delays, and frequency/ momentum shifts. Refraction cannot occur in this geometry 
and will be studied in Sec. V. The geodesic equations then read

$$
\begin{aligned}
& \frac{d x}{d t}=\frac{2 k \mp g_{a \gamma \gamma} \dot{a}}{2 \omega \pm g_{a \gamma \gamma} a^{\prime}},
\end{aligned}
$$

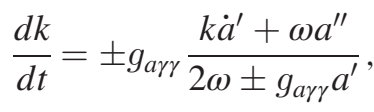

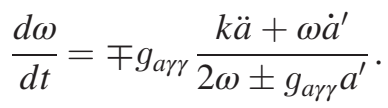

We now discuss the possibility of detecting spectral shifts when there is no axion profile at the end points of the trajectory. By perturbing about a background solution $\left(\omega_{0}, k_{0}\right)$, from Eqs. (30) and (31), we obtain the following expression for the frequency evolution up to $\mathcal{O}\left(g_{\text {ary }}^{2}\right)$,

$\frac{d \omega^{ \pm}}{d t}=\mp \frac{g_{a \gamma \gamma}}{2}\left[n_{0} \ddot{a}+\dot{a}^{\prime}\right]-\frac{g_{a \gamma \gamma}^{2} \partial_{t}(\partial a)^{2}}{4 k_{0}} \pm \mathcal{O}\left(g_{a \gamma \gamma}^{3}\right)$,

where

$$
n_{0}=\frac{k_{0}}{\omega_{0}}=\frac{k_{0}}{\left[k_{0}^{2}+\omega_{p}^{2}\right]^{1 / 2}}
$$

is the refractive index of the background plasma associated to the trajectory of an unperturbed ray. Note this is also equal to the unperturbed group velocity, $v_{g}^{0}=d x_{0} / d t=$ $k_{0} / \omega_{0}$, for a plasma background. Thus, by integration of (32), we have that

$$
\omega^{ \pm}(t)=\omega_{i}^{ \pm}+\delta \omega^{ \pm}(t),
$$

where $\omega_{i}^{ \pm}$is the initial frequency and

$$
\delta \omega^{ \pm}(t)= \pm \Delta \omega_{p}+\Delta \omega_{a} \pm \mathcal{O}\left(g_{a \gamma \gamma}^{3}\right)
$$

is the frequency variation, with

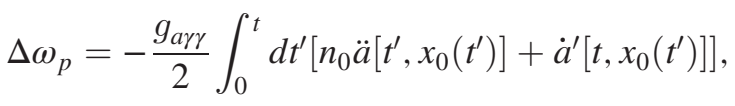

$$
\begin{aligned}
& \Delta \omega_{a}=-\frac{g_{a \gamma \gamma}^{2}}{4 k_{0}} \int_{0}^{t} d t^{\prime} \partial_{t}(\partial a)^{2},
\end{aligned}
$$

where $x_{0}(t)$ is the unperturbed photon trajectory-a straight line with constant phase and group velocity $v_{g}^{0}=d x_{0} / d t=k_{0} / \omega_{0}=n_{0}$,

$$
x_{0}(t)=v_{g}^{0} t+x_{i} .
$$

Note these are integrated frequency shifts, dependent on the whole evolution along the trajectory.
In the absence of plasma, the background refraction index is $n_{0}=1+\mathcal{O}\left(g_{\text {ary }}\right)$, so the first-order effect (36) reduces to

$$
\begin{aligned}
& \Delta \omega_{p} \rightarrow-\frac{g_{a \gamma \gamma}}{2} \int_{0}^{t} d t^{\prime}\left[\ddot{a}\left[t^{\prime}, x_{0}\left(t^{\prime}\right)\right]+\dot{a}^{\prime}\left[t, x_{0}\left(t^{\prime}\right)\right]\right] \\
& =-\frac{g_{\text {ary }}}{2}\left(\dot{a}\left(t, x_{0}(t)\right)-\dot{a}\left(0, x_{0}(0)\right)\right),
\end{aligned}
$$

where we have used the definition of Eulerian derivative, $d / d t=\partial_{t}+\left(d x_{0} / d t\right) \partial_{x}=\partial_{t}+\partial_{x}$, to write the integrand as a total derivative, thereby obtaining Eq. (16b) of Ref. [26]; the $\mathcal{O}\left(g_{\text {ary }}\right)$ result then becomes nonintegrated, depending only on the value of the axion field at the end points of the photon trajectory. Since we are investigating the case where there is no axion profile at the end points of the trajectory, the frequency shift (36) vanishes. In this case,

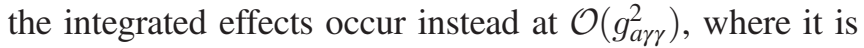
given by (37).

Furthermore, from the form of Eqs. (32), (36), and (37), it becomes apparent that one cannot simultaneously write both (36) and (37) as total derivatives when the background has both space and time dependence. Therefore, a localized axion profile can still impart a spectral shift onto the photon, even if it vanishes at the end points of the photon trajectory.

This is also apparent from simple kinematic arguments by considering a photon with initial/final frequencies and momenta $\left(\omega_{i}, \mathbf{k}_{i}\right)$ and $\left(\omega_{f}, \mathbf{k}_{f}\right)$, respectively, where the momentum transfer is provided by the axion background. If, asymptotically, the photon satisfies the axion-free massshell condition $\omega_{i, f}^{2}=\left(k_{i, f}^{2}+\omega_{p}^{2}\right)$, then an overall shift in frequency after passing through a local axion region must be accompanied by a shift in momentum, which requires both space and time translation symmetry to be broken. On the other hand, if the background has only one of space or time dependence, it is easy to see that, after a little algebra and using $x_{0}(t)=v_{g}^{0} t+x_{0}$, the integrands in (36) and (37) can be written as a total derivatives, so $\Delta \omega_{p}$ and $\Delta \omega_{a}$ depend only on the end points of the axion trajectory, preventing any asymptotic frequency shift for a localized axion background.

The former is a very important point. It means that a distant axion profile can impart integrated spectral shifts onto the photon, even if the profile vanishes at the asymptotic points of emission and detection. The different scenarios can be seen from the middle panel of Fig. 1. One especially interesting feature is that the frequency shift as measured at detection will modulate with the oscillation phase of the axion background, as shown in Fig. 2.

Clearly, it is also worth investigating whether any background which endows the photon with a nontrivial refractive index will lead to the same conclusions. This could have particular relevance for dielectric media in laboratory settings. 


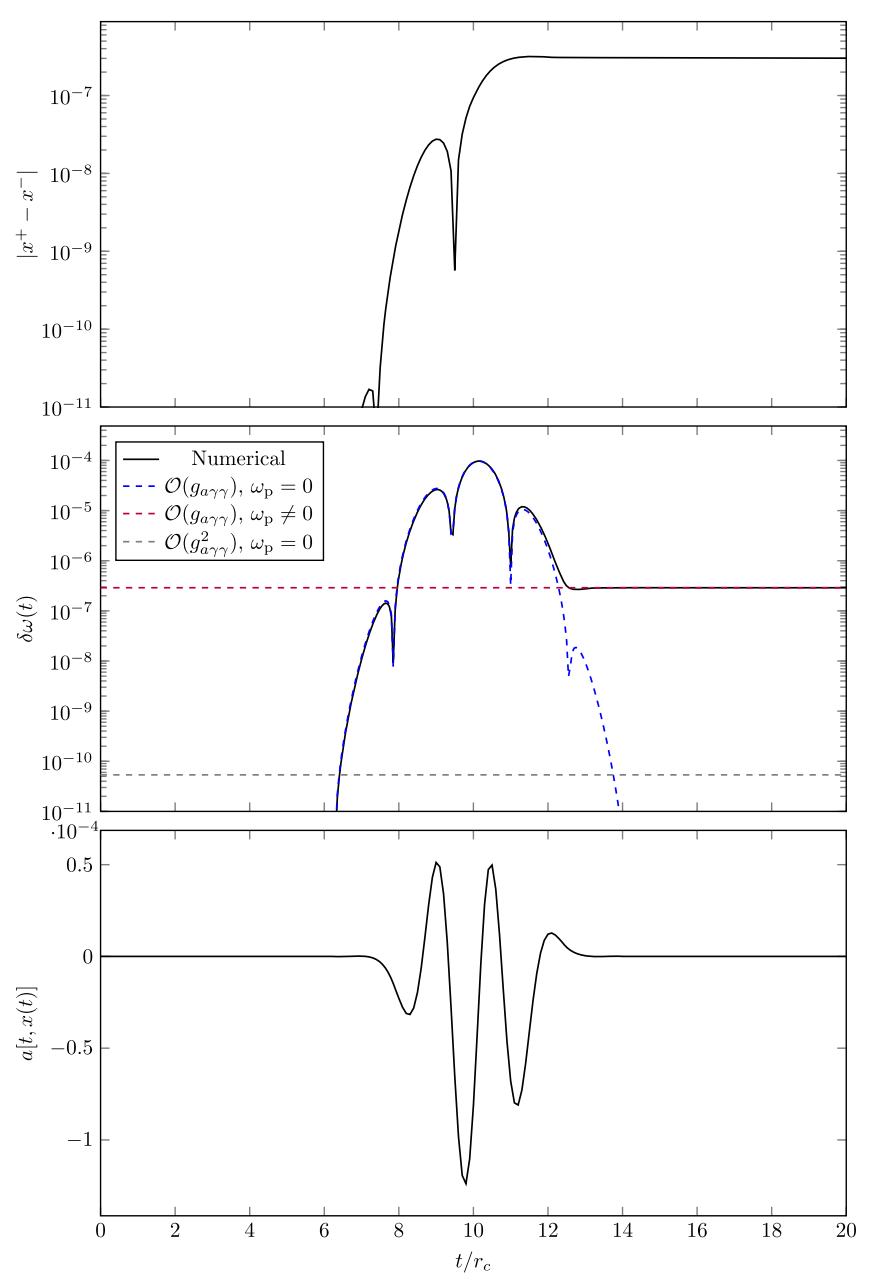

FIG. 1. Top panel.--Relative position/time splitting between left-right polarized photons in the presence of plasma. Middle panel.-Frequency shift for the left-polarized mode. We show the analytic results with and without plasma for comparison. The analytic results in the legend correspond to Eq. (39) (blue dashed) as well as the asymptotic frequency shifts from Eqs. (36) and (37) shown as the dashed horizontal lines. Bottom panel.-Axion profile evaluated along trajectory. We used the toy profile $a=$ $a_{0} \sin \left(m_{a} t\right) e^{-x^{2} / r_{c}^{2}}$ with $g_{a \gamma \gamma} a_{0}=10^{-4}, m_{a} r_{c}=0.5$ and $k_{0} r_{c}=5$. The plasma density is $\omega_{p} / k_{0}=0.08$, and the frequency and position are given in units with $r_{c}=1$.

\section{GROUP DISPERSION, BIREFRINGENCE, AND TIME DELAYS}

After extracting appropriate powers of $g_{\text {ary }}$ from all quantities involved, including momentum, we obtain the following expression for the group velocity perturbed about the zeroth-order trajectory

$$
\begin{aligned}
\frac{d x^{ \pm}}{d t}= & 1-\frac{\omega_{p}^{2}}{2 k_{0}^{2}} \pm \frac{g_{a \gamma \gamma} \omega_{p}^{2}\left(a^{\prime}-\dot{a}\right)}{2 k_{0}^{3}}+\frac{g_{a \gamma \gamma}^{2}(\partial a)^{2}}{8 k_{0}^{2}} \\
& \pm \frac{g_{a \gamma \gamma}^{3}(\partial a)^{2}\left[\dot{a}-a^{\prime}\right]}{8 k_{0}^{3}}+\mathcal{O}\left(g_{a \gamma \gamma}^{4}, \omega_{p}^{2} g_{a \gamma \gamma}^{2}\right) .
\end{aligned}
$$

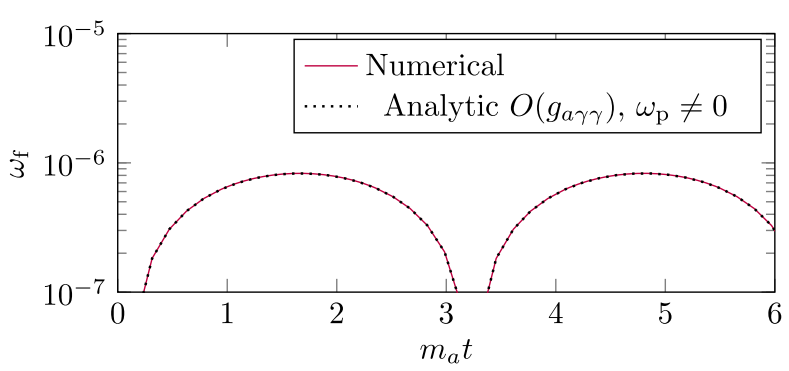

FIG. 2. Modulation of final frequency shift $\left|\omega_{f}\right|=\left|\omega(t)-\omega_{0}\right|$ at the point of detection for passage through a distant axion cloud which vanishes at the points of emission and detection. Other values are as for Fig. 1.

Note that there is a polarization-dependent group dispersion at $\mathcal{O}\left(g_{\text {ary }}\right)$ in the presence of plasma.

This can be seen in Fig. 1 numerically with a localized profile which vanishes at $x \rightarrow \pm \infty$. Since the onedimensional example contains no transverse gradients, photons travel in straight lines but with varying group velocity. Thus, the group arrival times are

$$
t=\int \frac{d t^{\prime}}{v_{g}\left(t^{\prime}\right)} .
$$

In the presence of a plasma, we find the following axion contribution to the time delay:

$$
\Delta t_{p}=\mp \frac{g_{a \gamma \gamma}}{4 k_{0}} \frac{\omega_{p}^{2}}{k_{0}^{2}} \int_{0}^{t_{f}} d t^{\prime}\left[a^{\prime}-\dot{a}\right] .
$$

This corresponds to the leading-order group velocity

$$
v_{g}=1-\frac{\omega_{p}^{2}}{k_{0}^{2}} \pm \frac{g_{a \gamma \gamma}}{4 k_{0}} \frac{\omega_{p}^{2}}{k_{0}^{2}}\left[a^{\prime}-\dot{a}\right]+\mathcal{O}\left(g_{a \gamma \gamma}^{2}\right) .
$$

The time delay is similar to that in a magnetized plasma, where the background magnetic field provides the breaking of isotropy, giving a group velocity [40]

$$
v_{g}=1-\frac{\omega_{p}^{2}}{k_{0}^{2}} \pm \frac{2 \omega_{p}^{2} \omega_{B}}{k_{0}^{3}}, \quad \omega_{B}=e|\mathbf{B}| / m_{e} .
$$

We note in passing, with an eye to future astrophysical applications, that the axion birefringent time delays can be distinguished from the magnetic version via the modulation of the axion signal due to axion oscillations, even though the two effects both run as $\propto 1 / k_{0}^{3}$.

Therefore, in the plasma case, $v_{g}<1$, so it seems reasonable to assume that $v_{g}$ can be treated as the speed of information transport, thus a meaningful observable for timing experiments. However, one should be cautious with 
identifying the group velocity with the speed of signal propagation in all instances, as we now discuss.

In the absence of plasma, one has $v_{g}(x, t) \simeq$ $1+g_{a \gamma \gamma}^{2}(\partial a)^{2} / 8 k_{0}^{2}$. Thus, for timelike field gradients, $\left(\partial_{\mu} a\right)^{2}>0$, we have $v_{g}>1$, i.e., a superluminal group velocity. This is in fact not forbidden [33,41]. However, in this case, the group velocity should not be interpreted as the speed at which information propagates [41,42]. Instead, the signal speed, rather than the group or phase velocity should be used for inferring the arrival of information $[33,43,44]$. The signal velocity derivation is more subtle and cannot be inferred in a simple way from the dispersion relation as for the phase and group velocity. Instead, it requires a more detailed treatment of the detection apparatus and the shape of the waveform to be detected [33]. We therefore leave such considerations for future work.

\section{REFRACTION}

We now discuss refraction of light rays passing through a localized axion profile. For this, it is sufficient to consider a $2+1$-dimensional background. If the photon is to experience deflection after passing through a localized axion profile, the outgoing phase velocity must have a different orientation than the incoming one.

\section{A. Refraction with plasma}

The leading-order correction to the momentum is

$$
\frac{d \mathbf{k}}{d t}= \pm \frac{g_{a \gamma \gamma}}{2}\left[n_{0} \nabla \dot{a}+\left(\hat{\mathbf{k}}_{0} \cdot \nabla\right) \nabla a\right]
$$

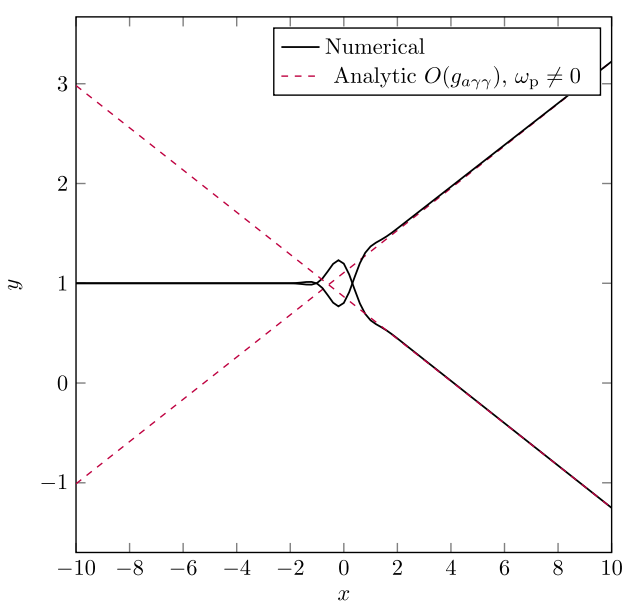

where we have used unperturbed solutions for $\omega$ and $\mathbf{x}$. When $n_{0} \neq 1$, the right-hand side is not a total derivative: upon integration, it is not simply given by the surface terms associated with the asymptotic field values at $x_{i}$ and $x_{f}$. This allows momentum changes through a localized axion background and therefore refraction to occur at $\mathcal{O}\left(g_{\text {ary }}\right)$ for nontrivial refractive indices. Integrating the momentum along the trajectory, the transverse component of the outgoing wave vector gives the deflection angle

$$
\sin \theta= \pm \frac{g_{a \gamma \gamma}}{2\left|\mathbf{k}_{0}\right|} \int_{0}^{t} d t^{\prime}\left[n_{0} \nabla_{\perp} \dot{a}+\left(\hat{\mathbf{k}}_{0} \cdot \nabla\right) \nabla_{\perp} a\right],
$$

where $\nabla_{\perp}$ is the gradient normal to the direction of initial propagation. Equation (46) shows that the existence of a plasma can induce polarization-dependent ray bending at leading order $\mathcal{O}\left(g_{\text {ary }}\right)$. Indeed, this will hold whenever the medium endows the photon with constant refractive index different from 1.

In the limit $\omega_{p}=0$, one has $n_{0}=1$, and this integral becomes a total derivative, just as shown in (39). This reproduces the conclusions of Ref. [26] that there is no chiral bending at $\mathcal{O}\left(g_{a \gamma \gamma}\right)$ for a pure-axion background.

We verified the validity of this approximation by solving the full geodesic equations in $2+1$ dimensions, the results of which are shown in Fig. 3.

\section{B. Refraction without plasma}

When the refractive index is equal to 1 , the momentum and frequency evolution are total derivatives at $\mathcal{O}\left(g_{\text {ary }}\right)$. Therefore, the nontrivial asymptotic shift in momentum is given at next-to-leading order,

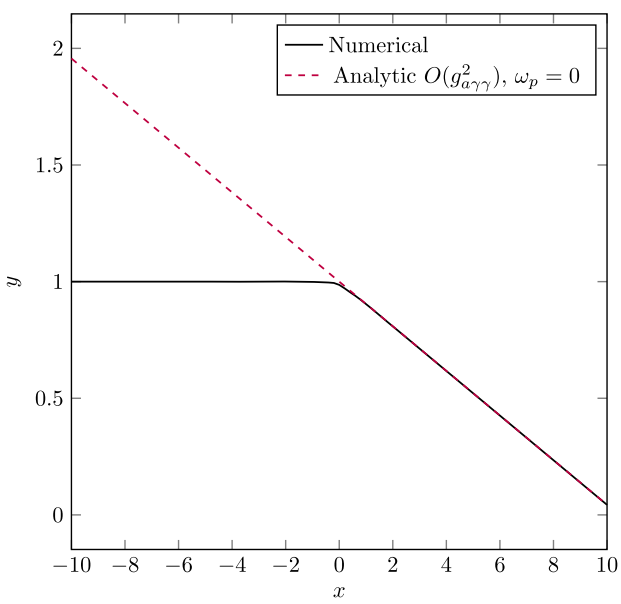

FIG. 3. Left panel.- Refraction through an axion cloud of left/right modes (black lines) with plasma, compared to the asymptotic trajectory (red dashed) predicted by Eq. (46). The black lines show the rays $(x(t), y(t))$ given by solving the geodesic equations (18)(20) numerically. The axes have been arbitrarily rescaled for visibility. We chose an impact parameter $b=r_{c}$ and a localized Gaussian axion profile, $a=a_{0} \sin \left(m_{a} t\right) \exp \left(-\left(x^{2}+y^{2}\right) / r_{c}^{2}\right)$, for illustration purposes. With $g_{a \gamma \gamma} a_{0}=5 \times 10^{-6}$ and $m_{a} r_{c}=0.3$, $k_{0} r_{c}=30$, and a plasma mass $\omega_{p} /\left|\mathbf{k}_{0}\right|=0.01$. Right panel.-Refraction though an axion background without plasma, $\omega_{p}=0$, with comparison to the asymptotic trajectory (red dashed) predicted by Eq. (48). The other values are the same. Rays propagate from the left to the right of the plot in both cases. 


$$
\delta \mathbf{k}(t)=-\frac{g_{a \gamma \gamma}^{2}}{8\left|\mathbf{k}_{0}\right|} \int_{0}^{t} d t^{\prime}\left(\mathbf{k}_{0}\left(\mathbf{k}_{0} \cdot \nabla\right)+\hat{\mathbf{k}}_{0}^{\perp} \nabla_{\perp}\right)\left[(\partial a)^{2}\right],
$$

where $\mathbf{k}_{0}^{\perp}$ is a unit vector normal to the initial vector. The angle of deflection is then given by

$$
\sin \theta=-\frac{g_{a \gamma \gamma}^{2}}{8\left|\mathbf{k}_{0}\right|^{2}} \int_{0}^{\infty} d t^{\prime} \nabla_{\perp}(\partial a)^{2} .
$$

The analytic and numerical results in this case are compared in Fig 3. We therefore conclude that bending of light does occur in axion backgrounds, but to leading order in perturbation at $\mathcal{O}\left(g_{\text {ary }}^{2}\right)$, which is nonchiral. Indeed, since the axion background is generically space and time dependent, there is no symmetry property to conserve group or phase velocities, and thus it is not only surprising but indeed inevitable that the rays should be deflected at some order in perturbation theory, as these results show.

\section{CONCLUSIONS}

In this work, we have examined the dispersive properties of axion backgrounds which lead to spectral distortions, time delays, and refraction of crossing light. We studied these effects by solving the geodesic equations of Hamiltonian optics, deriving both numerical and analytic results.

Building on the work of Blas et al. [26], we verified the following: (i) There is no $\mathcal{O}\left(g_{\text {ary }}\right)$ asymptotic deflection of light for pure axion backgrounds, but $\mathcal{O}\left(g_{\text {ary }}\right)$ birefringent deflection can occur for a nontrivial refractive index of the unperturbed background, induced here by the presence of plasma. (ii) In the absence of a nontrivial background refractive index, we found that $\mathcal{O}\left(g_{a \gamma \gamma}^{2}\right)$ refraction does occur. It is unsurprising that refraction should occur at higher order, since there is no symmetry principle to prevent it as the background is both time and space dependent with an inhomogeneous refractive index.
Perhaps the former effect, induced by the plasma, can have some implications in terms of reviving the idea of Ref. [25], where if a sufficiently dense plasma surrounds the black hole the refraction generated by a nontrivial axion profile could still be probed at first order in $g_{a \gamma \gamma}$. It is also worth considering the possibility of probing geodesic deviations from vacuum trajectories in laboratory settings as a means to probe local axion backgrounds. (iii) A nontrivial constant background refractive index, provided here by a homogeneous plasma, led to integrated frequency shifts in the photon, even for axion profiles localized along the line of sight—see (36) and (37) and Fig. 1. These results could have implications for timing delays and spectral distortions of astrophysical sources, since the effect is present even when the axion field vanishes at the trajectory end points. This is especially interesting in light of the fact that plasmas are ubiquitous in astrophysical settings.

Another possibility that we have not considered here is the tachyonic instability for dense axion fields/low frequency photons $[20,28,39,45,46]$, where, for one mode, the frequency becomes imaginary. This can lead to a relative intensity shift between the two modes as one is amplified relative to the other-i.e., dichroism [9].

\section{ACKNOWLEDGMENTS}

J. I. M. acknowledges the support of the Alexander von Humboldt foundation, and L. B. V. is supported by FCT Grant No. PD/BD/140917/2020 and by the Centro de Investigação e Desenvolvimento em Matemática e Aplicações (CIDMA) da Universidade de Aveiro Project No. UID/MAT/04106/2020. We thank João Rosa for stimulating discussions during the early stages of this work and for insightful comments on the manuscript. J. I. M. is especially grateful for hospitality from the physics department at the University of Aveiro during his stay. We also thank Valerie Domcke, Francesca Chadha-Day, Björn Garbrecht, and Graham Shore for useful conversations.
[1] R. D. Peccei and H. R. Quinn, Phys. Rev. Lett. 38, 1440 (1977).

[2] S. Weinberg, Phys. Rev. Lett. 40, 223 (1978).

[3] F. Wilczek, Phys. Rev. Lett. 40, 279 (1978).

[4] J. P. Conlon, J. High Energy Phys. 05 (2006) 078.

[5] P. Svrcek and E. Witten, J. High Energy Phys. 06 (2006) 051.

[6] J. Preskill, M. B. Wise, and F. Wilczek, Phys. Lett. 120B, 127 (1983).

[7] L. F. Abbott and P. Sikivie, Phys. Lett. 120B, 133 (1983).

[8] M. Dine and W. Fischler, Phys. Lett. 120B, 137 (1983).

[9] D. J. E. Marsh, Phys. Rep. 643, 1 (2016).
[10] L. Hui, J. P. Ostriker, S. Tremaine, and E. Witten, Phys. Rev. D 95, 043541 (2017).

[11] S. J. Asztalos et al. (ADMX Collaboration), Phys. Rev. Lett. 104, 041301 (2010).

[12] A. Caldwell, G. Dvali, B. Majorovits, A. Millar, G. Raffelt, J. Redondo, O. Reimann, F. Simon, and F. Steffen (MADMAX Working Group), Phys. Rev. Lett. 118, 091801 (2017).

[13] A. J. Millar, G. G. Raffelt, J. Redondo, and F. D. Steffen, J. Cosmol. Astropart. Phys. 01 (2017) 061.

[14] B. Majorovits and J. Redondo (MADMAX Working Group), in Proceedings, 12th Patras Workshop on 
Axions, WIMPs and WISPs (PATRAS 2016), Jeju Island, South Korea, 2016 (Hamburg, 2017), pp. 94-97.

[15] V. Anastassopoulos et al. (CAST Collaboration), Nat. Phys. 13, 584 (2017).

[16] I. G. Irastorza et al., J. Cosmol. Astropart. Phys. 06 (2011) 013.

[17] B. Döbrich (ALPS-II Collaboration), in Proceedings, 9th Patras Workshop on Axions, WIMPs and WISPs (AXIONWIMP 2013), Mainz, Germany, 2013 (2013).

[18] J. Redondo and A. Ringwald, Contemp. Phys. 52, 211 (2011).

[19] S. L. Adler, J. Gamboa, F. Mendez, and J. Lopez-Sarrion, Ann. Phys. (Amsterdam) 323, 2851 (2008).

[20] S. M. Carroll, G. B. Field, and R. Jackiw, Phys. Rev. D 41, 1231 (1990).

[21] D. Harari and P. Sikivie, Phys. Lett. B 289, 67 (1992).

[22] W. DeRocco and A. Hook, Phys. Rev. D 98, 035021 (2018).

[23] Y. Chen, J. Shu, X. Xue, Q. Yuan, and Y. Zhao, Phys. Rev. Lett. 124, 061102 (2020).

[24] S. Mohanty and S. N. Nayak, Phys. Rev. Lett. 70, 4038 (1993); 76, 2825 (1996).

[25] A. D. Plascencia and A. Urbano, J. Cosmol. Astropart. Phys. 04 (2018) 059.

[26] D. Blas, A. Caputo, M. M. Ivanov, and L. Sberna, Phys. Dark Universe 27, 100428 (2020).

[27] J. Alfaro, A. A. Andrianov, M. Cambiaso, P. Giacconi, and R. Soldati, Int. J. Mod. Phys. A 25, 3271 (2010).

[28] A. A. Andrianov and R. Soldati, Phys. Lett. B 435, 449 (1998).

[29] D. Espriu and A. Renau, Phys. Rev. D 85, 025010 (2012).
[30] D. Espriu and A. Renau, Int. J. Mod. Phys. A 30, 1550099 (2015).

[31] R. K. Sachs and A. M. Wolfe, Astrophys. J. 147, 73 (1967); Gen. Relativ. Gravit. 39, 1929 (2007).

[32] S. Weinberg, Phys. Rev. 126, 1899 (1962).

[33] P. W. Milonni, Fast Light, Slow Light and Left-Handed Light (Institute of Physics Publishing, Bristol, UK, 2005).

[34] A. Arvanitaki, S. Dimopoulos, S. Dubovsky, N. Kaloper, and J. March-Russell, Phys. Rev. D 81, 123530 (2010).

[35] A. Arvanitaki, M. Baryakhtar, and X. Huang, Phys. Rev. D 91, 084011 (2015).

[36] S. L. Detweiler, Phys. Rev. D 22, 2323 (1980).

[37] A. Khmelnitsky and V. Rubakov, J. Cosmol. Astropart. Phys. 02 (2014) 019.

[38] A. Caputo, L. Sberna, M. Frias, D. Blas, P. Pani, L. Shao, and W. Yan, Phys. Rev. D 100, 063515 (2019).

[39] M. Boskovic, R. Brito, V. Cardoso, T. Ikeda, and H. Witek, Phys. Rev. D 99, 035006 (2019).

[40] A. Suresh and J. M. Cordes, Astrophys. J. 870, 29 (2019).

[41] P. C. Peters, Am. J. Phys. 56, 129 (1988).

[42] G. Diener, Phys. Lett. A 223, 327 (1996).

[43] L. Brillouin, Wave propagation and group velocity (Academic Press, New York, London, 1960), Vol. 8.

[44] G. M. Shore, in Proceedings, Time and matter: International Colloquium on the science of time (TAM 2002): Venice, Italy, 2002 (Singapore, 2003), pp. 45-66.

[45] V. Domcke, Y. Ema, and K. Mukaida, J. High Energy Phys. 02 (2020) 055.

[46] V. Domcke, B. von Harling, E. Morgante, and K. Mukaida, J. Cosmol. Astropart. Phys. 10 (2019) 032. 